\title{
COMPETITIVE PROCESSES AND COMPARISON DIFFERENTIAL SYSTEMS
}

BY

\author{
G. S. LADDE
}

ABSTRACT. Sufficient conditions are given for stability and nonnegativity of solutions of a system of differential equations, in particular, of comparison differential equations. Finally, it has been shown that the comparison differential equations represent the mathematical models for competitive processes in biological, physical and social sciences.

1. Introduction. Since 1960, comparison differential equations have been successfully employed to investigate a variety of problems in a unified way, of differential equations in the context of Lyapunov's second method. The importance of this method lies in the fact that properties of the solutions of a system of differential equations can be inferred from corresponding properties of the solutions of a system of comparison equations. A systematic use of comparison equations, and its applications are demonstrated in a recent monograph [15]. In general, it is assumed that the comparison differential system possesses properties [13], [14], [15] in order to study the corresponding properties of solutions of a system of differential equations. However, in some special situations such properties have been examined [5], [7], .[8], [9] .

Based on our current study in biological, physical and social sciences, we immediately observe that the comparison systems represent models for competitive processes that exist in several fields, for instance, pharmocokinetics [2], [3], chemical kinetics [4] , [6], and economic systems [10], [11], [16], [18], [19] . This simple but very important observation opens up a new outlook towards the systems of comparison differential equations. Because of the significance of this observation, we propose to present three different aspects: (1) nonnegativity, (2) stability, and (3) interpretativity, of the comparison differential system.

In $\S 2$, we give sufficient conditions for nonnegativity of solutions of comparison systems, provided initial states of the systems are nonnegative. In $\$ 3$, sufficient conditions are given for stability of the equilibrium of a system of

Received by the editors June 25, 1975.

AMS (MOS) subject classifications (1970). Primary 34D20.

Key words and phrases. Chemical kinetics, comparison differential systems, compartment, competitive processes, concentration, economic systems, gross substitutability, Hicks matrix, interpretivity, maximal solution, Metzler matrix, nonnegativity, pharmacokinetics, quasimonotone property, reactant, stability. 
differential equations, in particular, that of the equilibrium of the comparison system. This stability analysis of the comparison system will justify the validity of the stability assumption made in [15], so as to study the stability behavior of a system of differential equations. Finally, in $\$ 4$, we interpret some of its mathematical conditions imposed on comparison systems to confirm that the comparison systems are indeed mathematical models for competitive processes in pharmacokinetics [2], [3], chemical kinetics [4], [6], and economic systems [10], [11], [16] , [18] , [19]. More detailed studies of these models are under investigation, and will appear elsewhere, see [20], [21].

2. Notations and definitions. Let $R^{n}$ denote the $n$-dimensional Euclidean space with any convenient norm $\|\cdot\|_{.} R_{+}$and $R$ stand for the nonnegative real and real line respectively. $R_{+}^{n}$ denotes the set $\left\{u \in R^{n}: u \geqslant 0\right\}$, that is (i.e.), a positive cone or positive orthant in $R^{n} . C\left[R_{+} \times R^{n}, R^{n}\right]$ denotes the space of continuous functions mapping the set $R_{+} \times R^{n}$ into $R^{n}$. Let $H_{i}=\left\{u \in R_{+}^{n}\right.$ : $\left.u_{i}=0\right\}$ for any $1 \leqslant i \leqslant n$.

Consider a system of differential equations

$$
\dot{u}=g(t, u), \quad u\left(t_{0}\right)=0,
$$

where $g \in C\left[R_{+} \times R^{n}, R^{n}\right]$, and $g(t, u)$ is smooth enough to assure the existence of solutions $u\left(t, t_{0}, u_{0}\right)$ of (1) for all $t \geqslant t_{0}, t_{0} \in R_{+}$.

3. Nonnegativity. In general, the solutions of systems of differential equations that describe systems in physical, biological, medical and social sciences, have to be nonnegative. Even in a mathematical point of view, it is interesting to derive sufficient conditions to establish the nonnegativity of solutions of a system of differential equations, especially, a comparison, or an auxiliary system of differential equations [15], that is most useful in studying the qualitative behavior of a system of differential equations in the context of Lyapunov's second method [15].

An attempt has been made by Hallam and Heidel [9] to study the nonnegativity of solutions for scalar comparison equations. In this section, we give sufficient conditions to establish the nonnegativity of solutions of a system of differential equations, whenever its initial data is nonnegative.

Here, we shall assume that $g(t, u)$ in (1) satisfies the following hypotheses:

$\left(\mathrm{NH}_{1}\right)$ Assume that $g(t, u)$ satisfies the following conditions

$$
g_{i}(t, u) \geqslant 0 \text { for all }(t, u) \in R_{+} \times H_{i}
$$

$$
\begin{aligned}
& \text { every (denote } \forall) i=1,2, \ldots, n . \\
& \left(\mathrm{NH}_{2}\right) \text { For }\left(t, u_{1}\right),\left(t, u_{2}\right) \in R_{+} \times R^{n} \\
& \quad\left\|g\left(t, u_{2}\right)-g\left(t, u_{2}\right)\right\| \leqslant L(t)\left\|u_{1}-u_{2}\right\|
\end{aligned}
$$


where $L \in C\left[R_{+}, R_{+}\right]$, i.e., $g(t, u)$ satisfies a Lipschitz condition in $u$ for fixed $t \in R_{+}$.

Now we prove the following:

THEOREM 1. Let the hypotheses $\left(\mathrm{NH}_{1}\right)$ and $\left(\mathrm{NH}_{2}\right)$ be satisfied. Let $u\left(t, t_{0}, u_{0}\right)$ be a solution of (1) through $\left(t_{0}, u_{0}\right) \in R_{+} \times R_{+}^{n}$. Then,

$$
u\left(t, t_{0}, u_{0}\right) \geqslant 0 \text { for } t \geqslant t_{0} \text {. }
$$

Proof. For sufficiently small $\mu \in R_{+}^{n} \backslash\{0\}$, consider the system of differential equations

$$
\dot{u}=g(t, u)+\mu, \quad u\left(t_{0}, \mu\right)=u_{0}+\mu, \quad u_{0} \geqslant 0 .
$$

It is easy to see that $g(t, u)+\mu$ satisfies all hypotheses of Theorem 2.5.2 in [15]. Therefore, the initial value problem (4) admits a unique solution $u(t, \mu)=$ $u\left(t, t_{0}, u_{0}, \mu\right)$ satisfying

$$
\lim _{\mu \rightarrow 0} u(t, \mu)=u\left(t, t_{0}, u_{0}\right), \quad t \geqslant t_{0},
$$

where $u\left(t, t_{0}, u_{0}\right)$ is the solution of (1) through $\left(t_{0}, u_{0}\right) \in R_{+} \times R_{+}^{n}$. The validity of the inequality (3) is immediate, if we can show that

$$
u(t, \mu)>0, \quad t \geqslant t_{0} .
$$

If (6) is false, let

$$
Z=\bigcup_{i=1}^{n}\left\{t \in R_{+}: u_{i}(t, \mu) \leqslant 0\right\}
$$

be a nonempty set, and $t_{1}=\inf Z$. Arguing as in Theorem 1.5.1 of [15], there exists index $j$ such that

(i) $u_{j}\left(t_{1}, \mu\right)=0$,

(ii) $u_{j}(t, \mu)>0, t \in\left[t_{0}, t_{1}\right)$,

(iii) $u_{i}(t, \mu) \geqslant 0, t \in\left[t_{0}, t_{1}\right], i \neq j$, and

$$
0 \geqslant \dot{u}_{j}\left(t_{1}, \mu\right)=g_{j}\left(t, u\left(t_{1}, \mu\right)\right)+\mu_{j} \text {. }
$$

This together with condition (2) in $\left(\mathrm{NH}_{1}\right)$ yields $0>\mu_{j}$ which is incompatible with the fact that $\mu \in R_{+}^{n} \backslash\{0\}$, i.e., $\mu_{j}>0$. Hence the set $Z$ is empty which in turn proves the inequality (6).

In the qualitative analysis of differential equations [15], $g(t, u)$ satisfies the quasimonotonicity property in $u$ for fixed $t \in R_{+}$, i.e.

$\left(\mathrm{NH}_{3}\right)$ for $u, v \in R_{+}^{n}$ such that $u_{i}=v_{i}, u_{k} \leqslant v_{k}$, for all $k \neq i, g(t, u)$ satisfies the relation

$$
g_{i}(t, u) \leqslant g_{i}(t, v), \quad \forall i=1,2, \ldots, n \text {. }
$$


$\left(\mathrm{NH}_{4}\right)$ Let us assume that the initial value problem (1) has a unique solution through $\left(t_{0}, u_{0}\right) \in R_{+} \times R_{+}^{n}$.

$\left(\mathrm{NH}_{5}\right)$ Assume that $g(t, u)=0$.

Remark 1. Observe that $\left(\mathrm{NH}_{3}\right)$ implies $\left(\mathrm{NH}_{1}\right)$ provided $g(t, 0)=0$; and $\left(\mathrm{NH}_{2}\right)$ implies $\left(\mathrm{NH}_{4}\right)$.

In the following, we state another result which establishes the nonnegativity of solutions of (1), whenever $\left(t_{0}, u_{0}\right) \in R_{+} \times R_{+}^{n}$.

THEOREM 2. Let the hypotheses $\left(\mathrm{NH}_{3}\right),\left(\mathrm{NH}_{4}\right)$ and $\left(\mathrm{NH}_{5}\right)$ be satisfied. Let $u\left(t, t_{0}, u_{0}\right)$ be a solution of $(1)$ through $\left(t_{0}, u_{0}\right) \in R_{+} \times R_{+}^{n}$. Then, $u\left(t, t_{0}, u_{0}\right)$ $\geqslant 0$ for $t \geqslant t_{0}$.

Proof. The proof of the theorem can be constructed by following the argument used in the proof of Theorem 1, except that the argument about continuous dependence of solutions with the parameter is replaced by the concept of the maximal solution of (1). Note that under the hypothesis $\left(\mathrm{NH}_{3}\right),(1)$ has a unique maximal solution.

REMARK 2. Assume that all the hypotheses of Theorem 2 hold except $\left(\mathrm{NH}_{4}\right)$. Then the conclusion of Theorem 2 is valid for the maximal solution of (1). Furthermore, Theorem 2 includes the result in [19] as a special case.

REMARK 3. From Theorems 1, 2 and Remark 1, we conclude that there is a trade-off between the hypotheses $\left(\mathrm{NH}_{1}\right),\left(\mathrm{NH}_{2}\right)$ and the hypotheses $\left(\mathrm{NH}_{3}\right)$, $\left(\mathrm{NH}_{4}\right)$.

In the following, we present some of the most useful special forms of (1). Consider the system of differential equations

$$
\dot{u}=A(t, u) w(t, u), \quad u\left(t_{0}\right)=u_{0},
$$

where $A$ is the $n \times n$ matrix function and $A \in C\left[R_{+} \times R^{n}, R^{n^{2}}\right], w(t, u)$ is the $n$-column vector function, and $w \in C\left[R_{+} \times R^{n}, R^{n}\right]$. Further assume that $A(t, u)$ and $w(t, u)$ are smooth enough to assure the existence of solutions of (8).

We assume that $A$ and $w$ in (8) satisfy the following hypotheses.

$\left(\mathrm{MH}_{1}\right)$ For $(t, u) \in R_{+} \times R_{+}^{n}$, the elements $a_{i j}(t, u)$ of the $n \times n$ matrix function $A(t, u)$ in (8) satisfy the relation

$$
a_{i i}(t, u)<0, \quad a_{i j}(t, u) \geqslant 0 \text { for } i \neq j, i, j=1,2, \ldots, n .
$$

Further assume that for $(t, u),(t, v) \in R_{+} \times R_{+}^{n}$, so that $u_{i}=v_{i}, u_{k} \leqslant v_{k}$ for all $k=1,2, \ldots, n$ and $k \neq i$, functions $a_{i i}(t, u), w_{i}(t, u), a_{i j}(t, u)$ satisfy the relations $a_{i i}(t, u) w_{i}(t, u) \leqslant a_{i i}(t, v) w_{i}(t, v), a_{i j}(t, u) \leqslant a_{i j}(t, v)$ for $j \neq i, \forall i$, $j=1,2, \ldots, n$, where $w_{i}(t, u)$ is the $i$ th component of the vector function $w(t, u)$ in (8).

$\left(\mathrm{MH}_{2}\right)$ The vector function $w(t, u) \geqslant 0, w(t, 0)=0$ and satisfies the quasi- 
monotonicity property in $u$ for fixed $t \in R_{+}$, where $(t, u) \in R_{+} \times R_{+}^{n}$.

$\left(\mathrm{MH}_{3}\right)$ Assume that the initial-value problem (8) has a unique solution through $\left(t_{0}, u_{0}\right) \in R_{+} \times R_{+}^{n}$.

REMARK 4. From $\left(\mathrm{MH}_{1}\right)$ and $\left(\mathrm{MH}_{2}\right)$, it is obvious that $g(t, u)=$ $A(t, u) w(t, u)$ satisfies the quasimonotonicity condition, i.e. the hypothesis $\left(\mathrm{NH}_{3}\right)$ and the hypothesis $\left(\mathrm{NH}_{5}\right) .\left(\mathrm{MH}_{3}\right)=\left(\mathrm{NH}_{3}\right)$ for $g(t, u)=A(t, u) w(t, u)$. With these observations, we immediately apply Theorem 2 , and conclude that any solution $u\left(t, t_{0}, u_{0}\right)$ of (8) is nonnegative, whenever $u_{0} \in R_{+}^{n}$.

From (8) several particular cases can be derived by replacing a pair $(A(t, u)$, $w(t, u))$ by its variant, i.e., any pair from the set

$$
\left\{\begin{array}{l}
(A(t, u), w(u)),(A(t, u), u),(A(u), w(t, u)),(A(u), w(u)),(A(u), u), \\
(A(t), w(t, u)),(A(t), w(u)),(A(t), u),(A, w(t, u)),(A, w(u)),(A, u)
\end{array}\right\} .
$$

REMARK 5. Note that a variant of a pair $(A(t, u), w(t, u))$ that satisfies $\left(\mathrm{MH}_{1}\right),\left(\mathrm{MH}_{2}\right)$, and $\left(\mathrm{MH}_{3}\right)$, will also satisfy these conditions. Hence Remark 3 is also applicable to them.

Recently, by using a method of successive approximations, Bellman [2] has proved the result concerning the nonnegativity of solutions of a special form $(A(u), u)$ of (8), under the condition (9) and assuming that the elements $a_{i j}(u)$ of $A(u)$ are Lipschitzian in $u \in R_{+}^{n}$. Furthermore, the variants $(A(t), u),(A, u)$ of (8) have been studied in [1], [2]. Thus our approach extends Bellman's results to a very general class of systems of differential equations.

REMARK 6. From the mathematical point of view the uniqueness assumption seems to be restrictive, but from the physical point of view, it is realistic. Moreover, in several cases, the function $g(t, u)$ in $(1)$ is at least once differential with respect to $u$ for fixed $t \in R_{+}$.

REMARK 7. In the pair $(A, u)$ in (10), the $n \times n$ matrix $A$ which satisfies (9), is called a Metzler matrix [16].

4. Stability. The stability properties of the solutions of a system of differential equations in the context of Lyapunov's second method [15] are inferred from corresponding stability properties of the solutions of a system of comparison differential equations [15]. However, the main problem in this method is determining under what conditions the comparison system possesses the desired stability behavior. An effort in this direction was made by Brauer [5], Grimmer [7], and Hallam and Heidel [9], Šiljak [17], Šiljak and Grujic [8] to establish the stability properties of scalar comparison differential equations.

In this section, we shall give sufficient conditions to establish the stability properties of the solutions of a system of differential equations, in particular, that of a comparison, or an auxiliary system of differential equations. We note 
that the system (1) satisfying the hypothesis $\left(\mathrm{NH}_{3}\right)$ is termed as a comparison, or an auxiliary system of differential equations. Similarly the system (8) and its variants (10) satisfying $\left(\mathrm{MH}_{1}\right)$ and $\left(\mathrm{MH}_{2}\right)$ are also termed as auxiliary, or comparison differential systems. Here and after, we shall use the term "auxiliary or comparison differential systems". In order to appreciate the stability conditions, and to appreciate the significance of the conditions, we shall limit our stability analysis to (8). However, based on our analysis, one can formulate more general conditions to study stability properties of (1).

In the following stability analysis, we shall consider stability in the sense of Lyapunov [15] of the equilibrium $u^{*}=0$ of the system (1). However, if $g\left(t, u^{*}\right)$ $=0$, all $t \in R_{+}$, and $u^{*} \neq 0$ is of interest, then we define the nonlinear function $g^{*}(t, v)=g\left(t, u^{*}+v\right)$, where $v^{*}=0$ represents the equilibrium $u^{*}$ under consideration. Further note that under the transformation $v=u-u^{*}, g^{*}(t, v)$ preserves the hypotheses $\left(\mathrm{NH}_{3}\right),\left(\mathrm{NH}_{4}\right)$ and $\left(\mathrm{NH}_{5}\right)$.

To derive a different set of sufficient conditions for stability of the equilibrium $u^{*}=0$ of (8), we shall assume that the functions $A(t, u), w(t, u)$ in (8) satisfy the following set of hypotheses:

$\left(\mathrm{SMH}_{1}\right)$ Assume that the elements $a_{i j}(t, u)$ of the $n \times n$ matrix function $A(t, u)$ and the components $w_{i}(t, u)$ of the vector function $w(t, u)$ in (8) satisfy the relations

$$
-\operatorname{sign}\left(a_{i i}(t, u) w_{i}(t, u)\right)=\operatorname{sign} u_{i}, \quad \text { all } t \in R_{+}
$$

and $a_{i j}(t, u)$ for $i \neq j$ may have mixed signs for $i, j=1,2, \ldots, n$; and

$$
\left|a_{j j}(t, u)\right|-d_{j}^{-1} \sum_{i=1 ; i \neq j}^{n} d_{i}\left|a_{i j}(t, u)\right| \geqslant \alpha_{j}(t, u),
$$

for all $(t, u) \in R_{+} \times R^{n}$, where $\alpha_{j} \in C\left[R_{+} \times R^{n}, R_{+}\right]$and $\alpha_{j}(t, \cdot) \in K\left[R^{n}\right]$ for each $t \in R_{+}$, i.e., $\alpha_{j}(t) \in C\left[R^{n}, R_{+}\right], \alpha_{j}(t, 0)=0$, all $t \in R_{+}, \alpha_{j}(t, u)$ is strictly increasing in $u$ for fixed $t \in R_{+}$; and $d_{j}>0, j=1,2, \ldots, n$. Further assume that $\alpha_{j}, w_{j}$ satisfy the conditions

$$
\left|w_{j}(t, u)\right| \alpha_{j}(t, u) \geqslant \phi_{j}(u)
$$

where $\phi_{j} \in K\left[R^{n}\right]$, i.e. $\phi_{j} \in C\left[R^{n}, R_{+}\right], \phi_{j}(0)=0, \phi_{j}(u)$ is strictly increasing in $u$. $\left(\mathrm{SMH}_{2}\right)$ For $(t, u) \in R_{+} \times R^{n}$, the elements $a_{i j}(t, u)$ of the matrix $A(t, u)$, and the elements of the vector functions $w(t, u)$ in (8) satisfy the relation (11), and further assume that

$$
\left|a_{i i}(t, u) w_{i}(t, u)\right| \geqslant \alpha_{i i}(t) \phi_{i}(u)
$$

and 


$$
\left|a_{i j}(t, u) w_{j}(t, u)\right| \leqslant \alpha_{i j}(t) \phi_{j}(u), \quad i \neq j,
$$

where $\phi_{j}$ are as defined in (13), $i, j=1,2, \ldots, n$; and the matrix function $C(t)$ defined by

$$
c_{i j}(t)= \begin{cases}-\alpha_{i i}(t), & i=j, \\ \alpha_{i j}(t), & i \neq j\end{cases}
$$

satisfies the relation

$$
\left|c_{j j}(t)\right|-d_{j}^{-1} \sum_{i=1 ; i \neq j}^{n} d_{i}\left|c_{i j}(t)\right| \geqslant \alpha, \quad t \in R_{+},
$$

for some $\alpha>0, d_{j}>0, j=1,2, \ldots, n$.

$\left(\mathrm{SMH}_{3}\right)$ Assume that the elements $a_{i j}(t, u)$ of the $n \times n$ matrix function $A(t, u)$, and the elements of the vector function $w(t, u)$ in (8) satisfy (11), and further assume that

$$
\left|a_{i i}(t, u)\right| \geqslant \alpha_{i i}(t), \quad\left|a_{i j}(t, u)\right| \leqslant \alpha_{i j}(t), \quad i \neq j
$$

and

$$
\left|w_{i}(t, u)\right| \geqslant \phi_{i}(u) \quad \text { for }(t, u) \in R_{+} \times R^{n},
$$

$i, j=1,2, \ldots, n$ where $\phi_{j}$ and $\alpha_{i j}$ are as defined in (14) and (15) and satisfy (17).

$\left(\mathrm{SMH}_{4}\right)$ Assume that the $\phi_{j}$ in $\left(\mathrm{SMH}_{1}\right)$, or $\left(\mathrm{SMH}_{2}\right)$, or $\left(\mathrm{SMH}_{3}\right)$ satisfy $\phi_{i}(u) \geqslant \epsilon u_{i}$ for $u_{i} \neq 0$ and for arbitrary small $\epsilon>0$.

$\left(\mathrm{SMH}_{5}\right)$ Assume that the hypothesis $\left(\mathrm{SMH}_{2}\right)$ or $\left(\mathrm{SMH}_{3}\right)$ holds except that the constant $\alpha$ is replaced by the function $\alpha(t)$ which satisfies

$$
\liminf _{t \rightarrow \infty}\left[\frac{1}{t-t_{0}} \int_{t_{0}}^{t} \alpha(s) d s\right]>0,
$$

and the functions $\phi_{j}$ satisfy $\left(\mathrm{SMH}_{4}\right)$.

REMARK 8. Note that the relation (11) in the hypotheses $\left(\mathrm{SMH}_{1}\right),\left(\mathrm{SMH}_{2}\right)$, $\left(\mathrm{SMH}_{3}\right)$, and $\left(\mathrm{SMH}_{5}\right)$ is equivalent to $a_{i i}(t, u)<0$, whenever $(t, u) \in R_{+} \times R_{+}^{n}$ and $w(t, u)$ satisfies $\left(\mathrm{MH}_{2}\right)$. In view of this fact we can conclude that the condition $a_{i i}(t, u)<0$ in $(9)$ is contained in these above mentioned hypotheses.

Now we prove the following.

THEOREM 3. Let the hypothesis $\left(\mathrm{SMH}_{1}\right)$ be satisfied. Then, the equilibrium $u^{*}=0$ of (8) is asymptotically stable.

Proof. Let $u\left(t, t_{0}, u_{0}\right)$ be a solution of (8) through $\left(t_{0}, u_{0}\right) \in R_{+} \times R^{n}$ existing for $t \geqslant t_{0}$. Let us consider the function $v: R^{n} \rightarrow R_{+}$defined by 


$$
v(u)=\sum_{i=1}^{n} d_{i}\left|u_{i}\right|
$$

where $d_{i}>0, i=1,2, \ldots, n$ as defined in (12). It is easy to observe that

$$
a(\|u\|) \leqslant v(u) \leqslant b(\|u\|), \quad(t, u) \in R_{+} \times R^{n},
$$

where $a(\|u\|)=d_{m}\|u\|, b(\|u\|)=\|d\|\|u\|, d_{m}=\min _{1<i<n} d_{i}$.

Now, by using (11), (12), (13), and (21), for $(t, u) \in R_{+} \times R^{n}$, we compute

$$
\begin{aligned}
D_{(8)}^{+} v(u) & =\sum_{i=1}^{n} d_{i} \operatorname{sign} u_{i} \dot{u}_{i} \\
& =\sum_{i=1}^{n} d_{i} \operatorname{sign} u_{i}\left[\sum_{j=1}^{n} a_{i j}(t, u) w_{j}(t, u)\right] \\
& =\sum_{i=1}^{n} d_{i}\left[\sum_{j=1}^{n} \operatorname{sign} u_{i} a_{i j}(t, u) w_{j}(t, u)\right] \\
& \leqslant \sum_{i=1}^{n} d_{i}\left[-\left|a_{i i}(t, u)\right|\left|w_{i}(t, u)\right|+\sum_{j=1 ; j \neq i}^{n}\left|a_{i j}(t, u)\right|\left|w_{j}(t, u)\right|\right] \\
& \leqslant \sum_{j=1}^{n} d_{j}\left|w_{j}(t, u)\right|\left[-\left|a_{j j}(t, u)\right|+d_{j}^{-1} \sum_{i=1 ; i \neq j}^{n} d_{i}\left|a_{i j}(t, u)\right|\right] \\
& \leqslant-\sum_{j=1}^{n} d_{j}\left|w_{j}(t, u)\right| \alpha_{j}(t, u) \\
& \leqslant-\sum_{j=1}^{n} d_{j} \phi_{j}(u) \leqslant-\phi(u)
\end{aligned}
$$

where

$$
\phi(u)=\sum_{j=1}^{n} d_{j} \phi_{j}(u) .
$$

Note that $\phi(u)$ is positive definite in $u$. Hence by the application of Lyapunov Theorem [12], we conclude that the equilibrium $u^{*}=0$ of (8) is asymptotically stable.

We formulate a few results which establish either asymptotic stability or exponential asymptotic stability of the equilibrium of (8).

THEOREM 4. Let the assumption $\left(\mathrm{SMH}_{2}\right)$ or $\left(\mathrm{SMH}_{3}\right)$ hold. Then the equilibrium $u^{*}=0$ of $(8)$ is asymptotically stable. 
Proof. The proof of the theorem can be constructed by imitating the proof of Theorem 3 with a little modification.

THEOREM 5. Let the hypotheses $\left(\mathrm{SMH}_{1}\right)$ and $\left(\mathrm{SMH}_{4}\right)\left(\left(\mathrm{SMH}_{2}\right)\right.$, or $\left(\mathrm{SMH}_{3}\right)$ and $\left.\left(\mathrm{SMH}_{4}\right)\right)$ be satisfied. Then the equilibrium $u^{*}=0$ is exponentially asympotically stable.

Proof. By imitating the proof of Theorem 3, we arrive at (23). From $\left(\mathrm{SMH}_{4}\right)$, for $u \neq 0$, and for an arbitrary small $\epsilon>0$ we have

$$
\phi_{i}(u) \geqslant \epsilon u_{i} \quad \text { for } i=1,2, \ldots, n \text {. }
$$

From (22), (23), (24) and (25) we obtain

$$
D_{(8)}^{+} v(u) \leqslant-\epsilon v(u) \quad \text { for }(t, u) \in R_{+} \times R^{n} .
$$

This together with (22) yields

$$
\left\|u\left(t, t_{0}, u_{0}\right)\right\| \leqslant K\left\|u_{0}\right\| \exp \left[-\epsilon\left(t-t_{0}\right)\right], \quad t \geqslant t_{0},
$$

where $K=d_{m}^{-1}\|d\|$. This completes the proof of the theorem.

THEOREM 6. Let the hypotheses $\left(\mathrm{SMH}_{5}\right)$ be satisfied. Then, the conclusion of Theorem 5 remains true.

REMARK 9. It is easy to observe that our stability results for the equilibrium of (8) include the stability results for the equilibrium of the comparison or the auxiliary systems of differential equations. In this case, one can easily see that the hypotheses $\left(\mathrm{SMH}_{1}\right)-\left(\mathrm{SMH}_{5}\right)$ need to satisfy on $(t, u) \in R_{+} \times R_{+}^{n}$ only. Therefore, the relation (11) is unnecessary in view of Remark 8.

REMARK 10. Based on previous results, one can formulate several stability results corresponding to the different set of conditions relative to variants (10) of (8). To avoid monotonicity, we are not going to formulate these conditions, however, we will present some of the conditions in order to show earlier results relative to comparison systems as special cases. For example: if we take the variant $(A, u)$ of $(8)$, i.e., $\dot{u}=A u$, that satisfies $\left(\mathrm{MH}_{1}\right)-\left(\mathrm{MH}_{3}\right)$. It is obvious that $\left(\mathrm{SMH}_{2}\right)$ holds with $\phi_{i}(u)=u_{i}$ and $\alpha_{i j}(t)=a_{i j}$, except that the relation (17) is supposed to be assumed relative to $A=C$. In this case the $n \times n$ constant matrix $A$ is a Metzler matrix [16]. It is well known that the Metzler matrix satisfying condition (17) implies it is a Hicks matrix [16] which implies that it is a stable matrix [16] that means that every eigenvalue of the matrix $A$ has negative real parts. This simple illustration has been utilized in [1], [17] in order to derive stability conditions for systems under investigation. Similarly, another case $(A, w(u))$, i.e. $\dot{u}=A w(u)$ can be studied based on conditions on $A$. This case has been utilized in [8] to investigate the stability of large-scale systems. 
5. Applications. In this section, we will interpret our conditions and hypotheses imposed on a comparison differential system (8) by means of following physical considerations. We present some of these applications in brief, however, more details about them are under investigation and will appear elsewhere, see [20], [21].

Pharmacokinetics. A compartmental system in pharmacokinetics consisting of $n$ interconnected compartments is described by the auxiliary system of differential equations (8). $u$ in (8) stands for the concentration of a certain drug or a chemical in the system. It is natural to expect that $u \in R_{+}^{n}$. The element $a_{i j}(t, u), i \neq j$, of the $n \times n$ matrix $A(t, u)$ in (8) characterizes the time and concentration dependent rates of transport material from compartment $j$ to compartment $i$ per unit amount of material in $j$ th compartment. $a_{i i}(t, u)$ is the total turnover rate of the $i$ th compartment. Because of their physical significance, the elements $a_{i j}(t, u)$ must satisfy the condition (9). The component $w_{j}(t, u)$ of $w(t, u)$ represents the law by means of which the material from the $j$ th compartment is transferred to $i$ th compartment, therefore, $w(t, u)$ has to be nonnegative vector. This fact justifies the assumption $\left(\mathrm{MH}_{2}\right)$. The condition $\left(\mathrm{MH}_{3}\right)$ reflects a unique response which is natural to expect. The condition $\left(\mathrm{SMH}_{2}\right)$ reflects the growth on the rate functions $a_{i j}(t, u)$.

To illustrate these facts with the system (8), we consider a four-compartmental model in pharmacokinetics [3]:

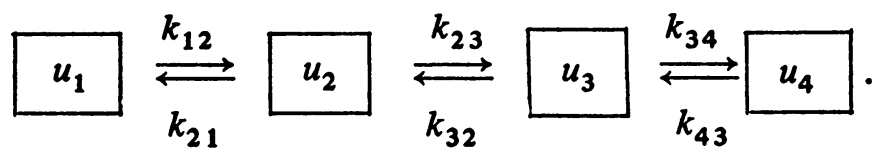

In this case we have the following system of equations

$$
\dot{u}=A u, \quad u\left(t_{0}\right)=u_{0} \geqslant 0
$$

where $A$ is a $4 \times 4$ constant matrix which is defined by

$$
A=\left[\begin{array}{cccc}
-k_{12} & k_{21} & 0 & 0 \\
k_{12} & -\left(k_{21}+k_{23}\right) & k_{32} & 0 \\
0 & k_{23} & -\left(k_{32}+k_{34}\right) & k_{43} \\
0 & 0 & k_{34} & -k_{43}
\end{array}\right]
$$

where $k_{i j}$ are rate constants in (27). It is obvious that (27) satisfies conditions $\left(\mathrm{MH}_{1}\right)-\left(\mathrm{MH}_{3}\right)$, and $\left(\mathrm{SMH}_{3}\right),\left(\mathrm{SMH}_{4}\right)$. If we assume that $A$ satisfies (17), then we can conclude from Theorem 5 that the equilibrium $u^{*}=0$ of (28) is exponentially asymptotically stable.

Chemical kinetics. The auxiliary differential system (8) may represent the 
interconversion of $n$ reactants in the chemical reaction system. Again $u$ represents the concentration vector. Obviously $u \in R_{+}^{n}$. The matrix $A(t, u)$ is the reaction rate matrix, and the vector $w(t, u)$ represents law by means of which the reactants convert into one another. The negativity of $a_{i i}(t, u)$ represents the self-inhibitory effect, and the $a_{i j}(t, u) \geqslant 0, i \neq j$, represents the activational effect of $j$ th reactant on the $i$ th reactant. The condition $\left(\mathrm{SMH}_{3}\right)$ again includes the saturation effects. Moreover, it is natural to expect that the rates do not grow infinitely.

We now illustrate these facts by a simple, monomolecular reversible open reaction system [6] described by the mechanism in which reactants $U_{1}$ and $U_{2}$ enter and leave the system at definite rates. This is represented by

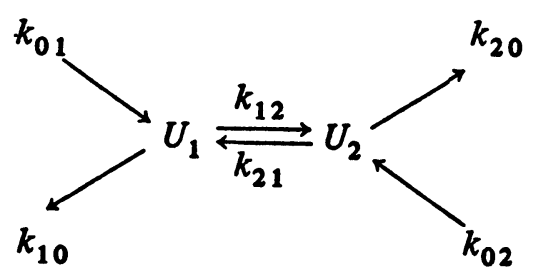

and the corresponding system of rate equations is

$$
\dot{u}=A u+k
$$

where $A$ is a $2 \times 2$ constant matrix defined by

$$
A=\left[\begin{array}{cc}
-k_{12} & k_{21} \\
k_{12} & -k_{21}
\end{array}\right]
$$

where $k_{i i}>0, k_{i j} \geqslant 0, i \neq j, i, j=1,2$, and

$$
k=\left[\begin{array}{l}
k_{01}-k_{10} \\
k_{20}-k_{20}
\end{array}\right] .
$$

Note that (30) satisfies the conditions $\left(\mathrm{MH}_{1}\right)-\left(\mathrm{MH}_{3}\right)$, and $\left(\mathrm{SMH}_{3}\right),\left(\mathrm{SHM}_{4}\right)$. If we assume that $A$ satisfies the conditions (17), then we can conclude that the steady-state $u^{*}$ of $(30)$ is exponentially asymptotically stable.

Economic systems. Comparison system (1) also represents the economic system in which $u$ is the price vector, the function $g(t, u)$ is the aggregate excess demand vector function. The assumption $\left(\mathrm{NH}_{3}\right)$ for $(1),\left(\mathrm{MH}_{1}\right)$ and $\left(\mathrm{MH}_{2}\right)$ for (8) reflects the time-dependent gross substitutability property [10], [11], [16], [19], i.e., the $j$ th commodity in a system of $n$ commodities is said to be a gross substitute for the $i$ th commodity if an increase in the price of the $j$ th commodity creates excess demand for the $i$ th commodity whose price has remained constant for $i \neq j, i=1,2, \ldots, n$. Relative to condition $\left(\mathrm{SMH}_{3}\right)$, we can make a remark similar to previous cases, such as they reflect a leveling-off of the influence of the 
$j$ th market price $u_{j}$ on the rate of change $\dot{u}_{i}$ of the $i$ th market price $u_{i}$, which is a realistic assumption on multiple-market system (1), or (8).

\section{REFERENCES}

1. R. Bellman, Introduction to matrix analysis, McGraw-Hill, New York, 1960. MR 23 \#A153.

2. - Topics in pharmacokinetics. I: Concentration dependent rates, Math. Biosci. 6 (1970), 13-17.

3. - Topics in pharmacokinetics. IV: Approximation in process space and fitting by sums of exponentials, Math. Biosci. 14 (1972), 45-47.

4. S. W. Benson, The foundations of chemical kinetics, McGraw-Hill, New York, 1960.

5. F. Brauer, Some refinements of Lyapunov's second method, Canad. J. Math. 17 (1965), 811-819. MR $31 \# 3670$.

6. H. G. Bray and K. White, Kinetics and thermodynamics in biochemistry, 2 nd ed., J. \& A. Churchill, London, 1966.

7. R. Grimmer, Stability of a scalar differential equation (to appear).

8. L. J. T. Grujić and D. D. Siljak, Asymptotic stability and instability of large-scale systems, IEEE Trans. AC-18 (1973), 636-645.

9. T. G. Hallam and J. W. Heidel, Structure of the solution set of some first order differential equations of comparison type, Trans. Amer. Math. Soc. 160 (1971), 501-512. MR 43 \#7709.

10. J. R. Hicks, Value and capital, 2nd ed., Oxford Univ. Press, Oxford, 1946.

11. L. A. Metzler, Stability of multiple markets: The Hicks conditions, Econometrica 13 (1945), 277-292. MR 7, 465.

12. K. K. Krasovskii, Certain problems in the theory of stability of motion, Fizmatgiz, Moscow, 1959; English transl., Stability of motion. Application of Ljapunov's second method to differential systems and equations with delay, Stanford Univ. Press, Stanford, Calif., 1963. MR 21 \#5047; 26 \#5258.

13. G. S. Ladde, Systems of differential inequalities and stochastic differential equations. II, J. Mathematical Phys. 16 (1975), 894-900.

14. - Variational comparison theorem and perturbations of nonlinear systems, Proc. Amer. Math. Soc. 52 (1975), 181-187.

15. V. Lakshmikantham and S. Leela, Differential and integral inequalities, theory and applications. Vol. I, Academic Press, New York, 1969.

16. P. K. Newman, Some notes on stability conditions, Rev. Econ. Studies 72 (1959), $1-9$.

17. D. D. Siljak, Stability of large-scale systems under structural perturbations,

IEEE Trans. Systems, Man and Cybernet. SMC-2 (1972), 657-663. MR 47 \#4669. 389-400.

18. - Connective stability of competitive equilibrium, Automatika 11 (1975),

19. K. J. Arrow and F. H. Hahn, General competitive analysis, Holden-Day, San Francisco, Calif., 1971. press).

20. G. S. Ladde, Cellular systems. I: Stability of chemical systems, Math. Biosci. (in (in press).

21. Cellular systems. II: Stability of compartmental systems, Math. Biosci.

DEPARTMENT OF MATHEMATICS, STATE UNIVERSITY OF NEW YORK, COLLEGE AT POTSDAM, POTSDAM, NEW YORK 13676 